\title{
Inverse Transform with Mellin like Feature
}

Benedict Irwin ${ }^{1}$

${ }^{1}$ University of Cambridge

June 3, 2020

Create a transform somewhat in analogy to the Mellin transform which to some extent extracts sequence coefficients.

$$
\mathcal{M}_{s}[f(x)](s) \approx \Gamma(s) \phi(-s)
$$

where

$$
f(x)=\sum_{s=0}^{\infty} \frac{(-1)^{s}}{s !} \phi(s) x^{s}
$$

instead consider a transform $\mathcal{I}[f(x)](s)$ such that

$$
\mathcal{I}[f(x)](s) \approx \Gamma(s) \chi(-s)
$$

where

$$
\frac{f^{-1}(x)}{x}=\sum_{s=0}^{\infty} \frac{(-1)^{s}}{s !} \chi(s) x^{s}
$$

and example, the function

$$
f(x)=x+x^{2}
$$

has inverse as series

$$
\begin{gathered}
f^{-1}(x)=x-x^{2}+2 x^{3}-5 x^{4}+\cdots \\
\frac{f^{-1}(x)}{x}=1-x+2 x^{2}-5 x^{3}+\cdots=\sum_{s=0}^{\infty} \frac{(-1)^{s}}{s !} C_{s} x^{s}=\sum_{s=0}^{\infty} \frac{(-1)^{s}}{s !} \frac{(2 s) !}{(s+1) !} x^{s}
\end{gathered}
$$

then

$$
\chi(s)=\frac{\Gamma(1+2 s)}{\Gamma(2+s)}
$$

then

$$
\begin{gathered}
\mathcal{I}\left[x+x^{2}\right](s)=\frac{\Gamma(s) \Gamma(1-2 s)}{\Gamma(2-s)} \\
x \mathcal{M}^{-1}\left[\mathcal{I}\left[x+x^{2}\right](s)\right](x)=f^{-1}(x)
\end{gathered}
$$




\section{Results}

Then it would seem that

$$
\begin{array}{r}
\mathcal{I}\left[2 x^{2}-\sqrt{x^{2}+4 x^{4}}\right](s)=\frac{\Gamma(s) \Gamma(1-2 s)}{\Gamma(1-s)} \\
\mathcal{I}[W(x)](s)=\Gamma(s)(-1)^{s} \\
\mathcal{I}[-W(-x)](s)=\Gamma(s) \\
\mathcal{I}\left[\frac{x}{1-x}\right](s)=\Gamma(s) \Gamma(1-s) \\
\mathcal{I}\left[\frac{1-\sqrt{1-4 x}-2 x}{2 x}\right](s)=\Gamma(s) \Gamma(2-s) \\
\mathcal{I}\left[\log \left(\frac{1}{x}\right)\right](s)=\Gamma(s-1) \\
\mathcal{I}\left[W\left(\frac{1}{x}\right)\right](s)=\Gamma(s-2) \\
\mathcal{I}\left[e^{x}-1\right](s)=\frac{\Gamma(s) \Gamma(1-s)^{2}}{\Gamma(2-s)} \\
\mathcal{I}[\log (x)](s)=(-1)^{1-s} \Gamma(s-1) \\
\mathcal{I}\left[\frac{1}{e^{x}-1}\right](s)=\frac{\pi \csc (\pi s)}{1-s} \\
\mathcal{I}\left[-x-W\left(-x e^{-x}\right)\right](s)=\Gamma(s) \zeta(s)
\end{array}
$$

Some more generalised ones

$$
\begin{array}{r}
\mathcal{I}\left[\log \left(x^{k}\right)\right](s)=\left(-\frac{1}{k}\right)^{1-s} \Gamma(s-1) \\
\mathcal{I}\left[W\left(\frac{1}{x^{k}}\right)\right](s)=k^{-1-1 / k+s} \Gamma\left(s-1-\frac{1}{k}\right) \\
\mathcal{I}\left[W\left(x^{k}\right)\right](s)=(-k)^{-1+1 / k+s} \Gamma\left(s-1+\frac{1}{k}\right) \\
\mathcal{I}\left[-\frac{x}{x+W\left(-e^{-x} x\right)}\right](s)=\frac{\Gamma(s) \Gamma(1-s)}{s} \\
\mathcal{I}\left[\frac{\mathcal{I}\left[-2 W\left(-\frac{\sqrt{x}}{2}\right)\right](s)=s^{2} \Gamma(s)}{1-W(e x / k)}\right](s)=k s \Gamma(1-s) \\
\mathcal{I}\left[-x^{k} W\left(\frac{x^{1-k}}{A}\right)\right](s)=-A x^{k s} \Gamma(s)
\end{array}
$$

As described in a previous article on here: It would appear that for the function

$$
f(x)=x^{m}+x, m>1
$$


we get a series

$$
g(x)=\sum_{n=0}^{\infty}\left(\begin{array}{c}
m n \\
n
\end{array}\right) \frac{(-1)^{n} x^{(m-1) n+1}}{(m-1) n+1}
$$

these then have a set of consistent, hypergeometric series explainable as

$$
g(x)={ }_{(m-1)} F_{(m-2)}\left(\left\{\frac{1}{m}, \frac{2}{m}, \cdots, \frac{m-1}{m}\right\} ;\left\{\frac{2}{m-1}, \cdots, \frac{m-2}{m-1}, \frac{m}{m-1}\right\} ;-\frac{m^{m} x^{m-1}}{(m-1)^{m-1}}\right) \cdot x
$$

then

$$
\frac{g(x)}{x}={ }_{(m-1)} F_{(m-2)}\left(\left\{\frac{1}{m}, \frac{2}{m}, \cdots, \frac{m-1}{m}\right\} ;\left\{\frac{2}{m-1}, \cdots, \frac{m-2}{m-1}, \frac{m}{m-1}\right\} ;-\frac{m^{m} x^{m-1}}{(m-1)^{m-1}}\right)
$$

which would give

$\mathcal{I}\left[x+x^{m}\right](s)=\mathcal{M}_{x}\left[{ }_{(m-1)} F_{(m-2)}\left(\left\{\frac{1}{m}, \frac{2}{m}, \cdots, \frac{m-1}{m}\right\} ;\left\{\frac{2}{m-1}, \cdots, \frac{m-2}{m-1}, \frac{m}{m-1}\right\} ;-\frac{m^{m} x^{m-1}}{(m-1)^{m-1}}\right)\right](s)$

which gives

$$
\begin{array}{r}
\mathcal{I}\left[x+x^{2}\right](s)=\frac{\Gamma(s / 1) \Gamma(1-2 s / 1)}{\Gamma(2-s)} \\
\mathcal{I}\left[x+x^{3}\right](s)=\frac{\Gamma\left(1-\frac{3 s}{2}\right) \Gamma\left(\frac{s}{2}\right)}{2 \Gamma(2-s)} \\
\mathcal{I}\left[x+x^{4}\right](s)=\frac{8 \cdot 3^{s-\frac{5}{2}} \pi \Gamma(-4 s / 3) \Gamma(s / 3)}{\Gamma(2 / 3-s / 3) \Gamma(4 / 3-s / 3) \Gamma(-s / 3)}=?=\frac{\Gamma(1-4 s / 3) \Gamma(s / 3)}{3 \Gamma(2-s)}
\end{array}
$$

it then seems like

$$
\frac{\Gamma\left(1-\frac{m s}{m-1}\right) \Gamma\left(\frac{s}{m-1}\right)}{(m-1) \Gamma(2-s)}=\mathcal{I}_{x}\left[x+x^{m}\right](s)
$$

and more generally

$$
\frac{a^{\frac{s}{m-1}} \Gamma\left(1-\frac{m s}{m-1}\right) \Gamma\left(\frac{s}{m-1}\right)}{(m-1) \Gamma(2-s)}=\mathcal{I}_{x}\left[x+a x^{m}\right](s)
$$

\section{Further Small polynomials}

There are some other small polynomials that give integer sequences upon reversion. Consider $x-x^{2}-x^{3}$.

$$
\mathcal{I}_{s}^{-1}[\Gamma(a+b s)](x)=(-1)^{b}(b+a)^{b} W\left(-\frac{b^{\frac{1}{b+a}} x^{\frac{1}{b+a}}}{b+a}\right)^{b}
$$

